\title{
What is the Retrospective Correlation between High- Grade Squamous Intraepithelial Lesion (HSIL) on Cytology and the Histological Diagnosis of Cervical Intraepithelial Neoplasia 2 (CIN2) or More in AML, Antwerp, Belgium?
}

\author{
Nina Karia ${ }^{* 1 \#}$, Alison Van Loon ${ }^{1 \#}$, Ina Benoy ${ }^{1,2,3}$ and Johannes Bogers ${ }^{1,2,3,4}$ \\ ${ }^{1}$ AMBIOR, Laboratory for Cell Biology \& Histology, University of Antwerp, Universiteitsplein 1, 2610 Antwerp, Belgium \\ ${ }^{2}$ Laboratory of Molecular Pathology, AML, Emiel Vloorsstraat 9, Antwerp, Belgium \\ ${ }^{3}$ National Reference Centre for HPV, Juliette Wytsmanstraat 14, Brussels, Belgium \\ ${ }^{4}$ International Centre for Reproductive Health, Ghent University, De Pintelaan 185, Ghent, Belgium \\ "Both authors contributed equally to this research article
}

\begin{abstract}
Introduction: Cervical cancer is a major worldwide health problem. Therefore, regular cervical screening in order to make an early diagnosis can help to prevent cervical cancer. The aim of this retrospective study is to evaluate the correlation between HSIL on cytology and histological CIN2+ in AML, Antwerp and to compare two liquid-based cytological techniques ThinPrep ${ }^{\circledR}$ LBC (TP) and SurePath ${ }^{\text {TM }}$ LBC (SP).

Methods: 120 women with a HSIL positive cytological smear from 2014 (ThinPrep $\AA$ LBC) and another 120 from 2010 (SurePath ${ }^{\text {TM }}$ LBC) were anonymously randomised out of the AML database, according to predefined in- and exclusion criteria. The Belgian Cancer Registry (CIB and CHP) and the AML database were consulted for histological and cytological data and the researched variables (doctor's speciality, age, HPV status, -genotypes and -persistence) of these 240 women. 184 women, with histological follow-up within one year, out of 240 were included. Statistical analysis was performed using Stata 15.1 (StataCorp, USA). P-values and Odds-ratios were calculated.

Results: The CIN2+/HSIL ratio of all included 184 subjects was $75.5 \%$ (95\%Cl=69.3-81.8). The found $\mathrm{CIN} 2+$ percentages for TP and SP, were $75.8 \%(95 \% \mathrm{Cl} 67.0-84.6)$ and $75.3 \%(95 \% \mathrm{Cl} 66.5-84.1)$ respectively. For all included subjects the variables hrHPV infection ( $\mathrm{p}=0,008 ; \mathrm{OR}=6.97)$ and HPV16 infection ( $\mathrm{p}=0.004 ; \mathrm{OR}=2.79)$ were statistically significant for having CIN2+ on histology.
\end{abstract}

Conclusions: The found CIN2+/HSIL ratio of $75.5 \%$ in AML, Antwerp is similar to the percentages found in worldwide laboratories. HSIL positive women who are HPV16+ or hrHPV + are at significant higher risk for invasive cervical disease. No statistically significant difference in CIN2+\% was found between the two LBC techniques TP and SP.

Keywords: High-grade squamous intraepithelial lesion • Cervical intraepithelial neoplasia grade 2 or more $\bullet$ Cervical cytology $•$ Cervical histology $\bullet$ Cytohistological correlation

\section{Introduction}

As cervical cancer is a major health problem, regular cervical screening to make an early diagnosis can help prevent cervical cancer, through identifying and treating pre-invasive cervical lesions. The yearly number of new cervical cancer diagnoses in Belgium was 640 in 2016 [1]. Concerning the prevalence of High-grade Squamous Intraepithelial Lesion (HSIL) cytology in 2017, 0.4\% of cervical smears in Flanders (North Belgium) has a HSIL outcome [2]. As found in our systematic review [3], the worldwide percentage of HSIL-positive women who have a histological result of Cervical Intraepithelial Neoplasia 2 or more $\left(\mathrm{CIN2}^{+}\right)$is $77.5 \%$. In AML (Algemeen Medisch Laboratorium), Antwerp, Belgium no research has been done on the correlation between cytological HSIL and the diagnosis of $\mathrm{CIN} 2^{+}$. Therefore, in this retrospective study this relationship is evaluated for AML collecting samples from Flanders, Belgium. The aim of our study is to evaluate the correlation between the cytological screening and histological outcome in the diagnosis of cervical cancer, more specifically the correlation between HSIL on cytology and histological CIN2+.

*Address for Correspondence: Nina Karia, University of Antwerp, Universiteitsplein 1, Antwerp, 2020, Belgium, Tel: 0032499416423, E-mail: Nina. Karia@student.uantwerpen.be

Copyright: (c) 2020 Karia N, et al. This is an open-access article distributed under the terms of the Creative Commons Attribution License, which permits unrestricted use, distribution, and reproduction in any medium, provided the original author and source are credited.

Received 19 January 2020; Accepted 03 February 2020; Published 10 February 2020
AML used to work exclusively with the BD SurePath ${ }^{\mathrm{TM}}$ (SP) liquid-based cytology (LBC) until June 2013, thereafter ThinPrep ${ }^{\circledR}$ (TP) LBC was used exclusively. In previous studies little research has been done regarding the differences of effectiveness between these two LBC tests. Therefore, in this study a comparison between these methods is made, using two cohorts.

It is relevant to know if the $\mathrm{CIN2}+\mathrm{HSIL}$ ratio for $A M L$ is high or low, as this would guide further policy concerning treatment and follow-up. If it is low, regarding the practical relevance for individuals, patients will be confronted with unnecessary physical complications, for example future pregnancy problems [4]. Looking at the practical relevance in the field of medicine, in this setting the focus is wrongly on a "healthy" population who do not need treatment. This conducts a faulty expenditure of medical sources. Concerning the theoretical relevance, this study would contribute to a more up-to-date scientific knowledge in the field of cervical screening. Finally mentioning relevance in public health, the overtreatment does not increase the "healthy life years" and brings more costs to the community [5].

The goal of our study was to evaluate the correlation between HSIL on cytology and histological CIN2+ in AML, Antwerp and to analyse the contribution of several variables (doctor's speciality, age, HPV status, -genotypes and -persistence) on the outcome (CIN2 ${ }^{+} / \mathrm{HSIL}$ ratio). Females living in Flanders with HSIL on LBC from AML were enrolled. A similar value to the value found in our literature search, was expected in AML [3]. Next to the main aim of our study, a second research question concerned the comparison between the two liquid-based cytological techniques (SurePath ${ }^{\mathrm{TM}}$ and ThinPrep ${ }^{\circledR}$ ), for the $\mathrm{CIN2}{ }^{+} / \mathrm{HSIL}$ ratio in AML. 


\section{Materials and Methods}

\section{Study design and study subjects}

The study design used is a retrospective observational cohort study. Two cohorts of HSIL positive women, retrieved from the anonymised AML database, were analysed. No control group was composed. For cohort one, women of 18 years and older with a cytological diagnosis of HSIL made by ThinPrep ${ }^{\circledR}$ LBC were enrolled. Conventional cytology and SurePath ${ }^{\text {TM }}$ LBC test were excluded. For cohort two, women of 18 years and older with a cytological diagnosis of HSIL made by SurePath ${ }^{\mathrm{TM}}$ LBC test were enrolled. So ThinPrep ${ }^{\circledR}$ LBC and conventional cytology were excluded. The full overview for the criteria of enrolment in a chronological order can be found in Figure 1. For both cohorts cytological samples from which the identity could not be retrieved, were excluded, for example anonymous samples from "Gezondheidszorg en hulpverlening aan prostituees" (GHAPRO) and anonymous samples within the framework of participation in scientific research. Also, women based abroad, for example women living in the Netherlands visiting a Belgian doctor, were excluded.

Concerning the number of study subjects, 120 females with HSIL were enrolled for each cohort. This number was obtained by the Survey System Sample Size Calculator $[6,7]$. For the sample size calculation, a confidence level of $95 \%$ and a confidence interval margin of $+/-10 \%$ were used. After applying the predefined exclusion criteria, a study population of 797 women for cohort one and 681 women for cohort two was obtained (Figure 1). After calculation, the needed sample size was at least 86 women for cohort one and 84 for cohort two. Note that following definition for inclusion was used: of the enrolled women, only those with a histological follow-up within one year were included. To make sure that ample patients could be included, 120 females with HSIL were enrolled for both cohorts (240 enrolled women in total). In our study, final exclusion or "lost in follow-up" was thus defined as the following: all enrolled women with cytological follow-up within one year or any kind of follow-up after more than one year. These defined criteria were used because, as defined in our research question, the outcome of this study is the correlation between HSIL on cytology and $\mathrm{CIN2}{ }^{+}$on histology. $\mathrm{CIN2}^{+}$is a tissue diagnosis, and thus needs to be made on a histological specimen. When follow-up only consists of a repeat cytology, this does not give us any information about any possible histological tissue diagnosis.

\section{Diagnostic tests}

For this study, no additional interventions were conducted. Although, included patients underwent two interventions in the past, more specifically a cytological test (with HPV co-testing) and a histological test.

Concerning the cytological test, cervical cells were collected using the Cervex-Brush ${ }^{\circledR}$ Combi (Rovers). After collection, cells were immediately transferred into a vial containing a fixative fluid and transported in room temperature to AML for further processing. Samples collected in 2010 were processed according to the BD SurePath ${ }^{\mathrm{TM}}$ method which used an ethanolbased fluid as preservative. Cervical cells of the women who were analysed in 2014, were processed according to the Hologic ThinPrep ${ }^{\circledR}$ method, a methanol based fixative fluid. Upon arrival in the lab, the samples were split into two parts. One part was used for HPV genotyping with the RIATOL qPCR test, the other part for the preparation and staining of the thin-layer slides. All slides were screened microscopically, using Focal Point Guided Screening (FPGS), by well-trained cytotechnicians and classified according to the Bethesda classification. This method implies a first cytological examination with automated computer guided microscopes, which analyses the PAP smear slides and selects 22 focal points based on segmentation, feature extraction and object classification. These focal points are provided for the second cytological examination [8].

All lab results were entered in a database which also contains patient demographics (age) and sample collection information (sampling date, specialization smear taker and collection medium).

The AML database was linked with two Belgian Cancer Registry databases, to find histological and cytological follow-up data from study participants who underwent a first cytological test at AML. The Belgian Cancer Registry provides two separate registers containing histological and/or cytological information. The first register is called the "Cancer In Belgium register" (CIB), which contains only histological information about all cervical cancers registered in Belgium. The second register is called the "CytoHistoPathological register" (CHP), which contains all results of all cytological and histological cervical samples examined in Belgium. We consulted both registers in the given order. Consulting the CHP was necessary because the CIB database only reports new cancer diagnoses. $\mathrm{CHP}$ in the contrary, contains all cytological and histological cervix sample

\section{a. Cohort 1 (ThinPrep $®$ LBC, 2014)}

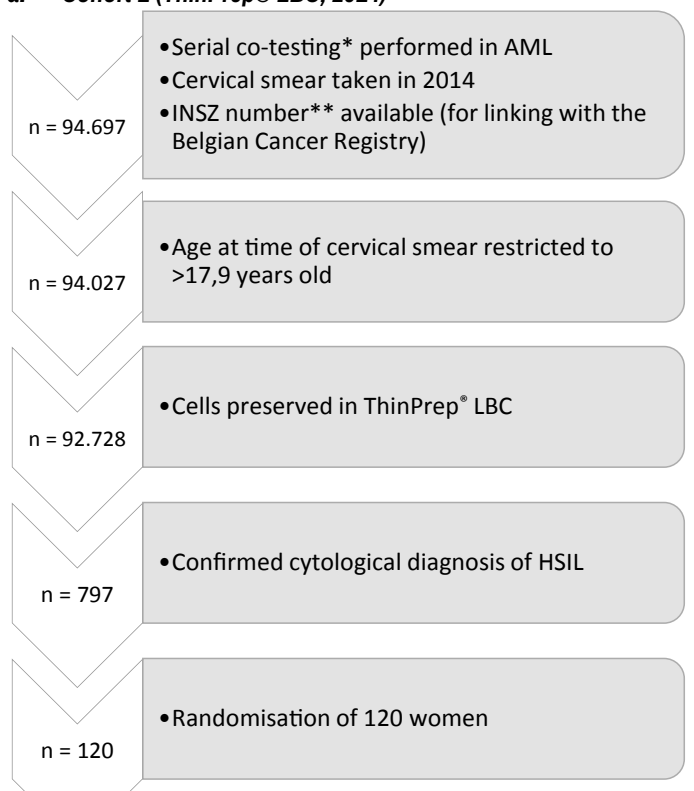

b. Cohort 2 (SurePath ${ }^{T M}$ LBC, 2010)

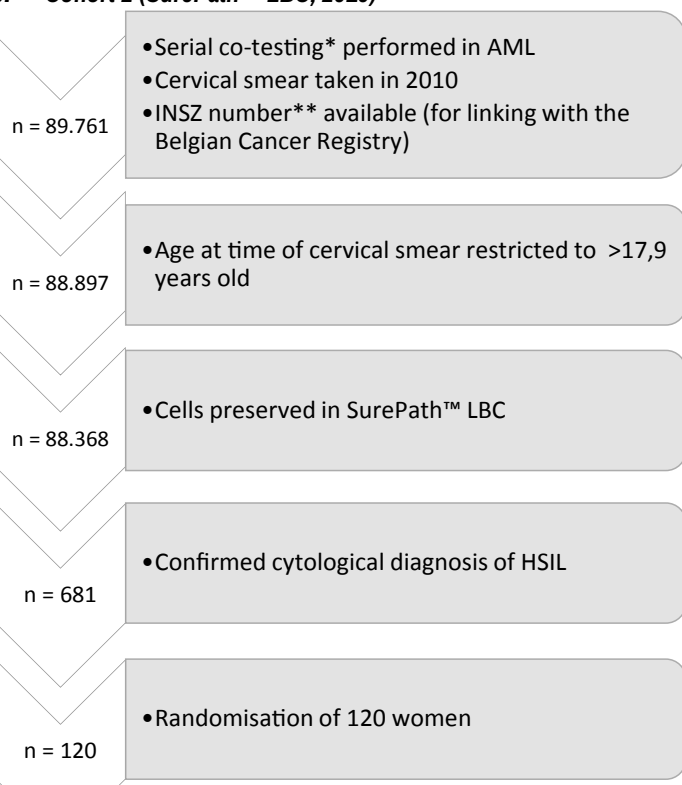

*Serial co-testing [6]: a unique algorithm for AML that positively influences the sensitivity of cytological screening, from $59,14 \%$ to $74,42 \%$. This means that at the time of cytological examination, there is foreknowledge of the HPV-status.

${ }^{* *}$ INSZ number $=$ Identification number of social security in Belgium

Figure 1. Flowcharts enrolment of subjects. 
results taken in Flanders. This means that cancer relapses will be described in CHP, but not in CIB. Thus, by solely consulting the CIB these would be missed as histological CIN2+ follow-up.

In practice, several histological methods are used after a cytological HSIL diagnosis, including colposcopically directed biopsy (CDB) from the most abnormal appearing area, large loop excision of the transformation zone (LLETZ), a large endocervical excision procedure (LEEP) and conisation [3]. In the consulted registers the used histological method was not mentioned.

In this study no extra intervention was conducted in addition to the abovementioned interventions. To conclude, we worked with the anonymised database of AML completed with the databases of the Belgian Cancer Registry, more specifically for Flanders the "Centrum voor Kankeropsporing" (CvKO).

Respecting the ethical considerations, approval of the Commission of Medical Ethics (UA-UZA) was obtained. The processing of personal data in pursuit of this study was limited to those data that are reasonably necessary to investigate the correlation between HSIL on cytology and histological $\mathrm{CIN2}^{+}$, in AML. These data were processed anonymously before statistical analysis with adequate precautions to ensure confidentiality. Researchers had no access to patient identification.

\section{Data collection}

Two random patient cohorts of 120 women each were enrolled using the excel randomisation function (Figure 1). For these 240 enrolled women, all data was retrieved from the AML database and the Belgian Cancer Registry CIB and CHP databases. The CIB database was always consulted first. After this, the CHP database and AML database were only consulted for those women who were not found in the CIB database. All data was anonymised during analysis, using a unique identification number. For both cohorts the information listed in Table 1 was retrieved in an anonymised manner from the AML database and Belgian Cancer Registry (CIB and CHP).

All the gathered information was collected into an overview excel spreadsheet. The elements histologic outcome, type of diagnostic intervention during follow-up and source of information were coded to make a statistical analysis, as further mentioned in Table 2a. Relevant variables for further statistical analysis were determined. The data concerning these variables in Table $2 b$ were coded. The used codes are mentioned in the last column of Table $2 \mathrm{~b}$. Before the statistical analysis was performed, the calculation of the proportion CIN2+/HSIL for cohort one, cohort two and the entire group of included subjects was made. $95 \%$ confidence intervals $(95 \% \mathrm{Cl})$ were calculated for these proportions, which equal $\beta+/-$ $z^{*} \sqrt{\left(\frac{\beta(1-\beta)}{n}\right)}$, where $\beta$ is the calculated proportion, $n$ is the sample size and $\mathrm{z}^{*}$ is the appropriate value from the standard normal distribution for a $95 \%$-confidence level (equalling 1.96).

\section{Statistics}

What concerns the statistical analysis of this study, a logistic regression was performed to evaluate the effect of different variables on the outcome (CIN2 ${ }^{+}$yes/no) and to investigate the effect of selection bias. Also, a multivariate regression was performed on the HPV types 16,31 and 67 , to investigate if one of these HPV types is a confounder. Statistical analysis was performed using Stata 15.1 (StataCorp, USA). P-values and Oddsratios with $95 \% \mathrm{Cl}$ were calculated. The obtained information was processed anonymously.

\section{Results}

In the following sections the found outcomes of each defined cohort are described separately, starting with cohort one (2014), followed by cohort two (2010). Afterwards, both cohorts are looked at together as one group and calculations are made for each of the abovementioned variables (see Methods). In the final section the results of the statistical analysis are described.

\section{Follow-up of the 2014 ThinPrep ${ }^{\circledR}$ cohort}

Analysing the 120 women from cohort one who had a TP test in 2014, we find that 64 women are found with follow-up in the CIB database, 44 women are found in the CHP and AML database and 12 women had no histological or cytological follow-up. From the 108 women that had undergone any follow-up, 102 had this follow-up within one year after their HSIL diagnosis. The other 6 women were excluded. 91 women of the remaining 102 women who had follow-up within 1 year, had histological follow-up and thus fit the inclusion criteria by our definition. When only looking at the women who had histological follow-up within 1 year after the cytological HSIL diagnosis, 91 of the 120 women remain. Identifying the $\mathrm{CIN2}{ }^{+}$results gives a number of 69 women who had a diagnosis of CIN2 or more on histology and 22 women who had CIN1 or less. Calculating the $\mathrm{CIN2}{ }^{+}$percentage gives a ratio of 69 over 91 with an outcome of $75.8 \%$. In Figure 2 an illustration can be found of the cohort from 2014, using TP test.

\section{Follow-up of the 2010 SurePath $^{\mathrm{TM}}$ cohort}

Analysing the 120 women from cohort two who had a SP test in 2010 , we find that 49 women are found with follow-up in the CIB database and 71 women are found in the CHP database. From the total of 120 women, 107 had follow-up within one year after their HSIL diagnosis. The other 13 women were excluded. 93 women of the remaining 107 women who had follow-up within 1 year, had histological follow-up and fit thus the inclusion criteria by our definition. So, when only looking at the women who had histological follow-up within 1 year after the cytological HSIL diagnosis, 93 of the 120 women remain. Identifying the CIN2+ results gives a number of 70 women who had a diagnosis of CIN2 or more on histology and 23 women who had CIN1 or less. Thus, calculating the $\mathrm{CIN} 2^{+}$percentage gives a ratio of 70 over 93 with an outcome of $75.3 \%$. In Figure 3 an illustration can be found of the cohort from 2010, using SP test.

\section{Outcome of cohort one and two}

The found CIN2+ percentages for cohort one and two were $75.8 \%$ and $75.3 \%$ respectively. 91 out of 120 and 93 out of 120 women with a HSILdiagnosis on cytology from cohort one and two had histological follow-up within one year. This is 184 out of 240 women. So, $76.7 \%$ of the HSIL positive women went for a biopsy within one year of their abnormal smear, and $23.3 \%$ were lost in follow-up.

Table 1. Retrieved data per source.

\begin{tabular}{|c|c|c|}
\hline AML database & CIB registry & CHP registry \\
\hline - Date of HSIL diagnosis & - Date of cervical histology & - Date of cervical histology or cytology \\
\hline - Age at the time of HSIL diagnosis & $\begin{array}{l}\text { - Outcome of histology according to the CIN } \\
\text { classification }\end{array}$ & $\begin{array}{l}\text { - Type of diagnostic intervention carried out (cytology } \\
\text { or histology) }\end{array}$ \\
\hline $\begin{array}{l}\text { - HPV status and genotypes at the time of HSIL } \\
\text { diagnosis }\end{array}$ & $\bullet$ Country code & $\begin{array}{c}\text { - Outcome of the carried out histological or cytological } \\
\text { test }\end{array}$ \\
\hline$\bullet$ Duration of the HPV infection & - Organ code & $\bullet$ Organ code \\
\hline \multicolumn{3}{|l|}{ - Specialty of the doctor who did the smear test } \\
\hline - Collection medium & & \\
\hline
\end{tabular}


Table 2. Description and coding of elements and variables,

a. Elements

\begin{tabular}{|c|c|c|}
\hline Element & Possible responses & Coded response \\
\hline \multirow{3}{*}{$\begin{array}{l}\text { Outcome of histology according to the } \mathrm{CIN} \\
\text { classification }\end{array}$} & $\bullet$ CIN2- & 0 \\
\hline & - CIN2+ & 1 \\
\hline & - Lost in follow-up according to definition* & 9 \\
\hline \multirow[t]{3}{*}{ Type of diagnostic intervention carried out } & $\bullet$ Histology & 1 \\
\hline & - Cytology & 2 \\
\hline & - No follow-up** & 3 \\
\hline \multirow[t]{3}{*}{ Source of information } & $\bullet \mathrm{CIB}$ & 1 \\
\hline & $\bullet \mathrm{CHP}$ & 2 \\
\hline & - AML & 3 \\
\hline
\end{tabular}

b. Variables

\begin{tabular}{|c|c|c|c|}
\hline Variable & Type & Possible responses & Coded response \\
\hline \multirow{2}{*}{$\begin{array}{l}\text { Cervical histology or cytology result available } \\
\text { within one year after HSIL diagnosis }\end{array}$} & \multirow[t]{2}{*}{ Dichotomous } & - No follow-up within one year & 0 \\
\hline & & • Follow-up within one year & 1 \\
\hline \multirow[t]{2}{*}{ Collection medium } & \multirow[t]{2}{*}{ Categorical } & - BD SurePath ${ }^{\mathrm{TM}}$ liquid-based Pap test & 1 \\
\hline & & $\bullet$ ThinPrep $^{\circledR}$ LBC & 2 \\
\hline \multirow[t]{2}{*}{ Doctor's specialty who did the smear test } & \multirow[t]{2}{*}{ Categorical } & - Gynaecologist & 1 \\
\hline & & - General practitioner & 2 \\
\hline \multirow[t]{2}{*}{ Age at the time of HSIL diagnosis } & \multirow[t]{2}{*}{ Dichotomous } & $\bullet<30$ years old & 0 \\
\hline & & - 30 years old or more & 1 \\
\hline \multirow[t]{2}{*}{ HPV status at the time of HSIL diagnosis } & \multirow[t]{2}{*}{ Dichotomous } & $\bullet \mathrm{HPV}$ - & 0 \\
\hline & & - HPV + & 1 \\
\hline \multirow[t]{2}{*}{ hrHPV status at the time of HSIL diagnosis } & \multirow[t]{2}{*}{ Dichotomous } & • hrHPV - & 0 \\
\hline & & $\bullet$ hrHPV + & 1 \\
\hline \multirow[t]{2}{*}{ IrHPV status at the time of HSIL diagnosis } & \multirow[t]{2}{*}{ Dichotomous } & •IrHPV - & 0 \\
\hline & & - IrHPV + & 1 \\
\hline \multirow{4}{*}{$\begin{array}{l}\text { Number of different hrHPV types at the time of } \\
\text { HSIL diagnosis }\end{array}$} & \multirow[t]{4}{*}{ Categorical } & $\bullet 0$ & 0 \\
\hline & & $\bullet 1$ & 1 \\
\hline & & $\bullet 2$ & 2 \\
\hline & & - more than 2 & 3 \\
\hline \multirow{2}{*}{$\begin{array}{c}\text { HPV type } 16 \text { status at the time of HSIL } \\
\text { diagnosis }\end{array}$} & \multirow[t]{2}{*}{ Dichotomous } & $\bullet$ HPV16 - & 0 \\
\hline & & $\bullet$ HPV16 + & 1 \\
\hline \multicolumn{4}{|c|}{ Analogous strategy as described above for HPV type 16 for following HPV types: $18,31,33,35,39,45,51,52,53,56,58,59,66,67,68$} \\
\hline \multirow[t]{3}{*}{$\begin{array}{l}\text { Persistence of HPV infection at the time of } \\
\text { HSIL diagnosis }\end{array}$} & \multirow[t]{3}{*}{ Categorical } & $\begin{array}{l}\text { - The present one and the same HPV type infection has not been present } \\
\text { for at least two years or absence of HPV infection }\end{array}$ & 0 \\
\hline & & $\begin{array}{l}\text { - The present one and the same HPV type infection has been present for } \\
\text { at least two years }\end{array}$ & 1 \\
\hline & & $\bullet$ Unknown & 9 \\
\hline
\end{tabular}

*Lost in follow-up = all women with HSIL cytology and cytological follow-up within one year or any kind of follow-up after more than one year.

${ }^{* *}$ No follow-up = all women with HSIL cytology with no cytological nor histological follow-up at any given time.

hrHPV = high risk HPV types, these are the following: $16,18,31,33,35,39,45,51,52,56,58,59,66$ and 68 ; IrHPV = low risk HPV types, these are the following 6,11 , 53 and 67

\section{Outcome of all included subjects applying the variables}

Opposed to the above paragraph, in this section we combine both cohorts to consider the entire group of included subjects, not separating the women in the two cohorts of 2014 and 2010. Thus, the 91 included women from 2014 and the 93 included women from 2010 are looked at together. For this entire group of 184 women a global CIN2+ percentage was calculated, what represents the main aim of our study.

After this, we identified diverse subgroups. We further calculated the percentages of $\mathrm{CIN}_{2}{ }^{+}$for these different variable subgroups, next to the global CIN2 or more percentage.

For this entire group of 184 women we found that $75.5 \%$ have $\mathrm{CIN2}{ }^{+}$ on histology within one year, with a range of $66.5 \%-78.5 \%$ and a $95 \% \mathrm{Cl}$ of $69.29 \%-81.72 \%$ (Table 3).
To point out, some women with a HSIL positive cervical smear do not undergo a biopsy but have a repeat cytology as follow-up. In the Methods section the inclusion criteria are mentioned which state that these women are excluded because cytology cannot give certainty about tissue diagnosis. In order to tackle this uncertainty a range was established, as seen in Table 3. The "range" consists of a lower limit and an upper limit. The lower limit is defined when hypothetically cytological follow-up within one year is also included as a valid follow-up and these cytological follow-up results are automatically identified as CIN2 negative on biopsy. The upper limit is defined when hypothetically cytological follow-up within one year is included as a valid follow-up and these cytological follow-up results are automatically identified as CIN2 positive on biopsy.

In Table 4 an overview of the calculation of the percentages of CIN2 for the analyzed variables can be found. When looking at the two subgroups 


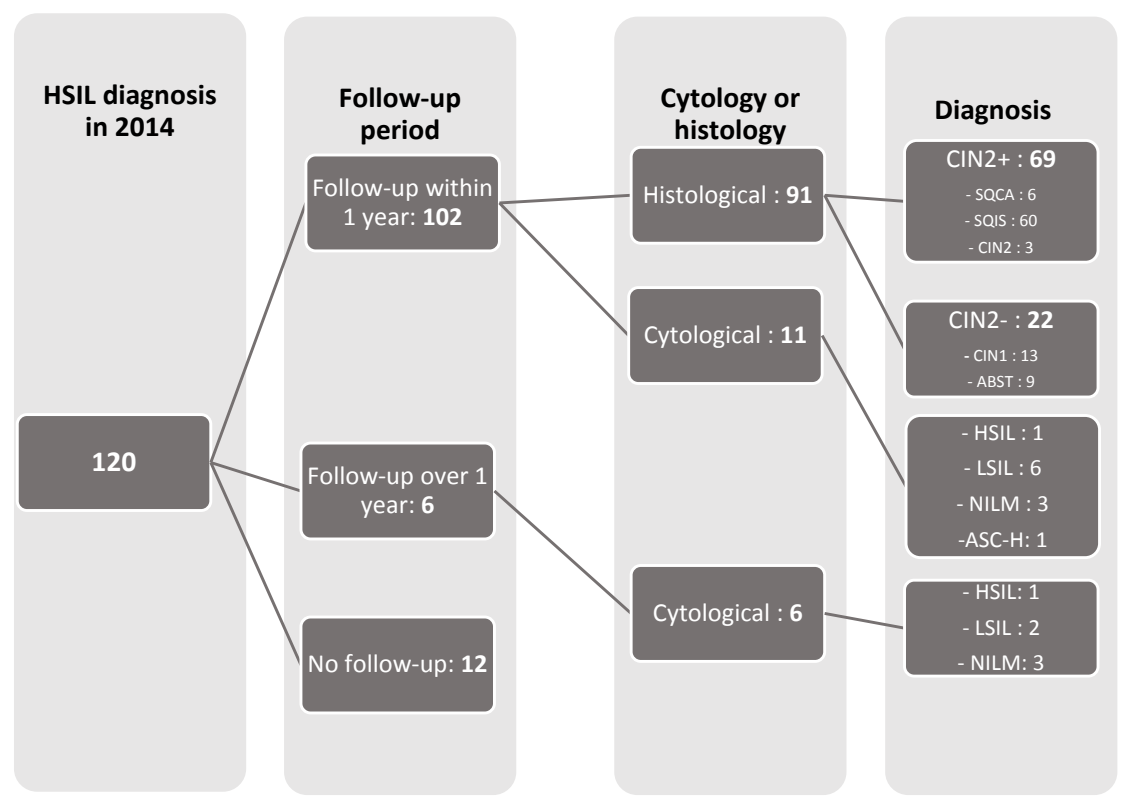

SQCA= Invasive squamous carcinoma, SQIS= Squamous carcinoma in situ, ABST= No dysplasia, nor tumor, HSIL= High-grade squamous intraepithelial lesion, LSIL= Low-grade squamous intraepithelial lesion, NILM= negative for intraepithelial lesion or malignacy, ASC-H= Atypical squamous cells, cannot exclude HSIL

Figure 2. Visual analytics cohort 1.

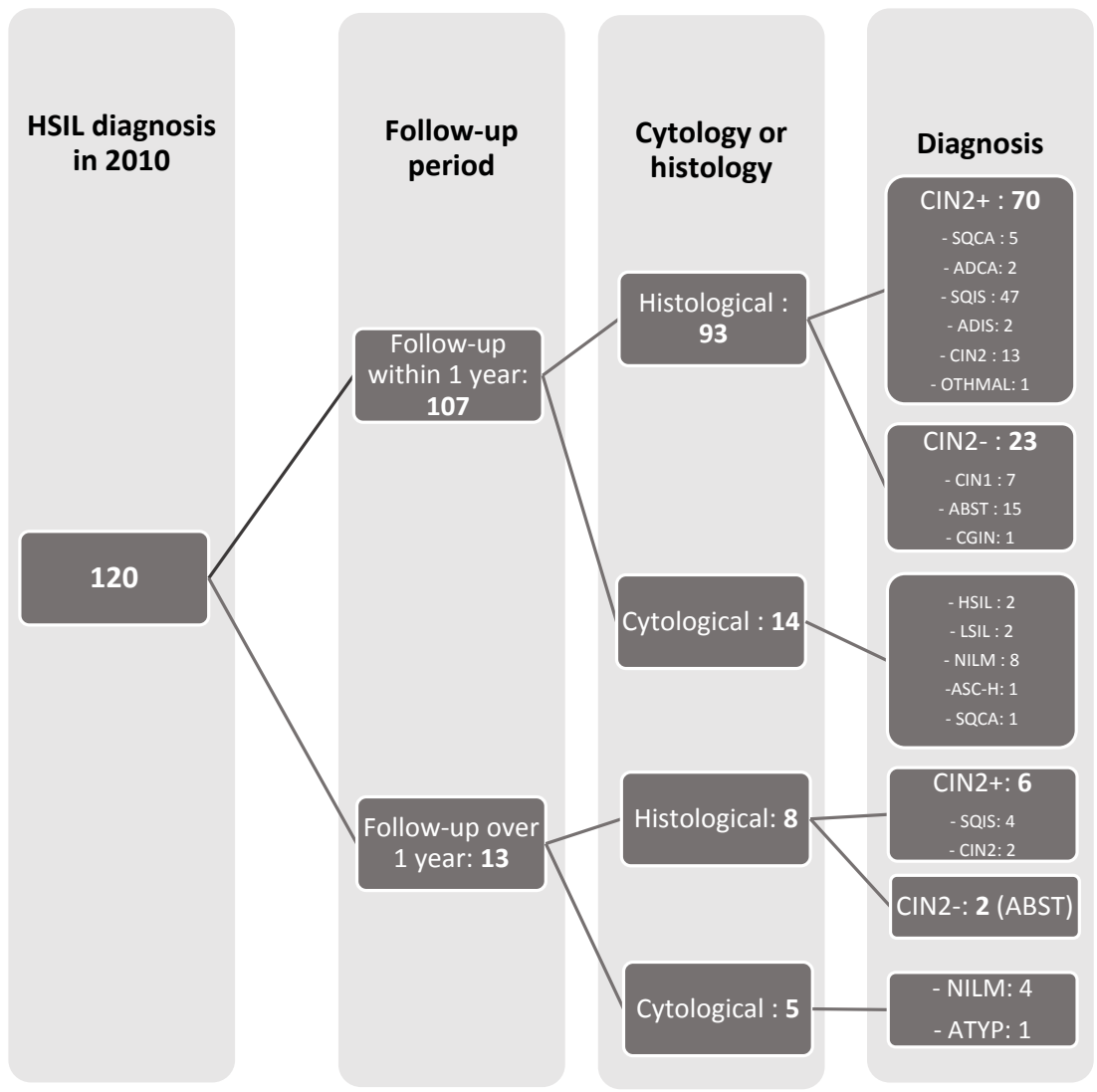

$A D C A=$ Invasive adenocarcinoma; $A D I S=$ Adenocarcinoma in situ; OTHMAL = Other malignancies, specified: tumors including metastasis and invasion of the cervix, other than ADIS, SQIS, ADSQIS, ADCA, SQCA, ADSQC; CGIN = Cervical Glandular Intraepithelial Neoplasia; ATYP = Atypia, not further specified

Figure 3. Visual analytics cohort 2.

of collection medium, there is a difference of $0.5 \%$ in $\mathrm{CIN} 2^{+}$percentage between both subgroups. There is a difference of $0.8 \%$ in $\mathrm{CIN} 2^{+}$percentage between the subgroups of doctor specialty. In the subgroups of age, there is a difference of $2.5 \%$ in $\mathrm{CIN} 2+\%$.

A difference of $44.4 \%$ in $\mathrm{CIN2} \%$ is seen between the subgroups of hrHPV status. $95.1 \%$ of all included women are hrHPV+. When looking at the subgroups of number of different HPV types, following percentages are found for respectively $0,1,2$, more than 2 number of hrHPV types: $33.3 \%$,
$78.7 \%, 75.5 \%, 78.1 \%$. For the subgroups of IrHPV, a difference of $13.6 \%$ is found, with $12 \%$ of all included women who are IrHPV+. Concerning the HPV types $16,18,31,33,35,39,45,51,52,53,56,58,59,66,67$, and 68 , a difference of respectively $18.5 \%, 7.4 \%, 8.4 \%, 9.5 \%, 9.5 \%, 6.8 \%, 24.9 \%$, $8.9 \%, 3.6 \%, 15.3 \%, 17.4 \%, 17.3 \%, 6.7 \%, 4.3 \%, 33.9 \%$ and $26.1 \%$ is noted in CIN2\% between the genotype positive and negative subgroups. When looking at the subgroups of persistence of HPV infection, this was unknown in $77.2 \%$ of the women. Between the other two subgroups (persistent 
Table 3. Main outcome.

\begin{tabular}{cccccccc}
\hline & \# included & CIN2- & CIN2+ & $\begin{array}{c}\text { CIN2+l } \\
\text { HSIL (\%) }\end{array}$ & 95\%Cl (\%) & Range (\%) & P-value \\
\hline All included subjects & 184 & 45 & 139 & 75.5 & $69.3-81.8$ & $66.5-78.5$ \\
\hline $\begin{array}{c}\text { Collection medium: TP } \\
\text { (cohort 1) }\end{array}$ & 91 & 22 & 69 & 75.8 & $67.0-84.6$ & $67.7-78.4$ \\
\hline $\begin{array}{c}\text { Collection medium: SP } \\
\text { (cohort 2) }\end{array}$ & 93 & 23 & 70 & 75.3 & $66.5-84.1$ & $65.4-78.5$
\end{tabular}

$A D C A=$ Invasive adenocarcinoma; $A D I S=$ Adenocarcinoma in situ; OTHMAL = Other malignancies, specified: tumors including metastasis and invasion of the cervix, other than ADIS, SQIS, ADSQIS, ADCA, SQCA, ADSQC; CGIN = Cervical Glandular Intraepithelial Neoplasia; ATYP = Atypia, not further specified

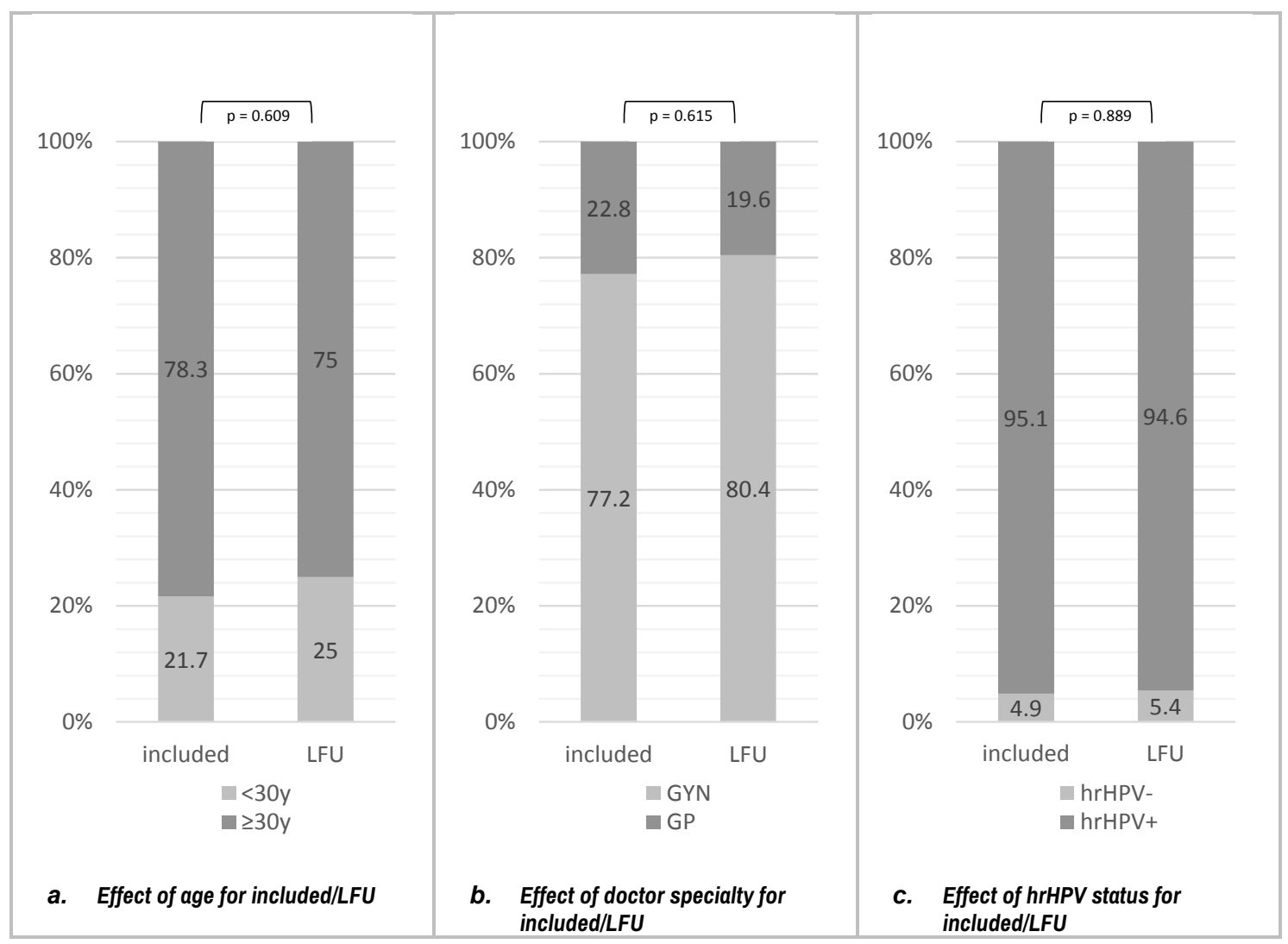

LFU = lost in follow-up; GYN = gynaecologist; GP = general practitioner

Figure 4. Examples of nonsignificant variables (lost in follow-up).

infection for at least two years and no persistent infection) there is a difference of $16 \%$ in $\mathrm{CIN} 2 \%$.

\section{Statistical analysis}

In this section the outcome of the statistical analysis with Stata 15.1 is described. It is divided into three segments: statistical analysis of the whole group of 184 included women, the first cohort from 2014 and the second cohort from 2010 consecutively.

Previous to these analyses on the 184 included women, a statistical analysis was performed on the 240 women enrolled after randomisation. No statistically significant variables were found to enhance the risk of being lost in follow-up (as defined in Table 2). These 56 women (23.3\%) had no significant predicting features for not having histological follow-up within one year. In particular nor age $(p=0.609)$, nor doctor's specialty $(p=0.615)$, nor hrHPV positivity $(p=0.889)$ influenced having histological follow-up within one year after HSIL diagnosis as shown in Figure 4.

\section{Statistical analysis of all 184 included women}

Of all 184 included women, those with a hrHPV infection at the time of HSIL diagnosis had 6.97 more chance (i.e. Odd's ratio, OR) to be $\mathrm{CIN2}{ }^{+}$ on histology than those who were hrHPV negative at that moment, with a $95 \%$-confidence interval $(95 \% \mathrm{Cl})$ of $1.67-29.17$. This is statistically significant $(\mathrm{p}=0.008)$ as in Table 5 and Figure 5.

A second statistically significant variable found for the whole group was HPV16 infection at the time of HSIL diagnosis. More specifically, of all 184 included women, those with a HPV16 infection had 2.79 more chance (OR) to be $\mathrm{CIN2}{ }^{+}$on histology than those who were HPV16 negative, with a $95 \% \mathrm{Cl}$ of $1.38-5.66(\mathrm{p}=0.004)$ (Table 5 and Figure 5 ).

Following variables were proven not to be statistically significant: the other researched HPV types $(18,31,33,35,39,45,51,52,53,56,58,59$, 66, 67 and 68), persistent HPV-infection as per definition, number of hrHPV types, IrHPV type, age and doctor specialty. Also, the variable "collection medium", the comparison between cohort one (TP) and two (SP), was again proven to be a not statistically significant variable (Table 3$)(p=0.930, O R$ $=1.03$ ).

The multivariate regression performed on the HPV types 16, 31 and 67 , showed that there is no confounding as the statistical significance of the different HPV types stays unchanged with this analysis. HPV type 16 continues to be statistically significant with $p=0.006$ and OR 2.76. HPV type 31 and 67 are still not significant (HPV31: $p=0.201$ and OR=1.90; HPV67: $\mathrm{p}=0.157$ and $\mathrm{OR}=0.32$ ). 
Table 4: Overview of the calculation of the percentages of $\mathrm{CIN} 2+$ for the analyzed variables

\begin{tabular}{|c|c|c|c|c|c|}
\hline Variable & \# included (\%) & CIN2- & CIN2+ & $\begin{array}{l}\text { CIN2+l } \\
\text { HSIL (\%) }\end{array}$ & P-value \\
\hline Doctor specialty: gynaecologist & $142(77.2)$ & 35 & 107 & 75.4 & 0.912 \\
\hline Doctor specialty: general practitioner & $42(22.8)$ & 10 & 32 & 76.2 & \\
\hline Age at the time of HSIL diagnosis: $<30 y$ & $40(21.7)$ & 9 & 31 & 77.5 & 0.745 \\
\hline Age at the time of HSIL diagnosis: $30 \mathrm{y}$ or more & $144(78.3)$ & 36 & 108 & 75 & \\
\hline hrHPV status at the time of HSIL diagnosis: - & $9(4.9)$ & 6 & 3 & 33.3 & 0.008 \\
\hline hrHPV status at the time of HSIL diagnosis: + & $175(95.1)$ & 39 & 136 & 77.7 & \\
\hline IrHPV status at the time of HSIL diagnosis: - & $162(88)$ & 37 & 125 & 77.2 & 0.172 \\
\hline IrHPV status at the time of HSIL diagnosis: + & $22(12)$ & 8 & 14 & 63.6 & \\
\hline Number of different hrHPV types at the time of HSIL diagnosis: 0 & $9(4.9)$ & 6 & 3 & 33.3 & 0.264 \\
\hline Number of different hrHPV types at the time of HSIL diagnosis: 1 & $94(51)$ & 20 & 74 & 78.7 & \\
\hline Number of different hrHPV types at the time of HSIL diagnosis: 2 & $49(26.6)$ & 12 & 37 & 75.5 & \\
\hline Number of different hrHPV types at the time of HSIL diagnosis: more than 2 & $32(17.5)$ & 7 & 25 & 78.1 & \\
\hline HPV type 16 status at the time of HSIL diagnosis: - & $88(47.8)$ & 30 & 58 & 65.9 & 0.004 \\
\hline HPV type 16 status at the time of HSIL diagnosis: + & $96(52.2)$ & 15 & 81 & 84.4 & \\
\hline HPV type 18 status at the time of HSIL diagnosis: - & $168(91.3)$ & 40 & 128 & 76.2 & 0.510 \\
\hline HPV type 18 status at the time of HSIL diagnosis: + & $16(8.7)$ & 5 & 11 & 68.8 & \\
\hline HPV type 31 status at the time of HSIL diagnosis: - & $150(81.5)$ & 39 & 111 & 74 & 0.310 \\
\hline HPV type 31 status at the time of HSIL diagnosis: + & $34(18.5)$ & 6 & 28 & 82.4 & \\
\hline HPV type 33 status at the time of HSIL diagnosis: - & $172(93.5)$ & 41 & 131 & 76.2 & 0.463 \\
\hline HPV type 33 status at the time of HSIL diagnosis: + & $12(6.5)$ & 4 & 8 & 66.7 & \\
\hline HPV type 35 status at the time of HSIL diagnosis: - & $172(93.5)$ & 41 & 131 & 76.2 & 0.463 \\
\hline HPV type 35 status at the time of HSIL diagnosis: + & $12(6.5)$ & 4 & 8 & 66.7 & \\
\hline HPV type 39 status at the time of HSIL diagnosis: - & $161(87.5)$ & 38 & 123 & 76.4 & 0.477 \\
\hline HPV type 39 status at the time of HSIL diagnosis: + & $23(12.5)$ & 7 & 16 & 69.6 & \\
\hline HPV type 45 status at the time of HSIL diagnosis: - & $181(98.4)$ & 45 & 136 & 75.1 & omitted \\
\hline HPV type 45 status at the time of HSIL diagnosis: + & $3(1.6)$ & 0 & 3 & 100 & \\
\hline HPV type 51 status at the time of HSIL diagnosis: - & $160(87)$ & 41 & 119 & 74.4 & 0.346 \\
\hline HPV type 51 status at the time of HSIL diagnosis: + & $24(13)$ & 4 & 20 & 83.3 & \\
\hline HPV type 52 status at the time of HSIL diagnosis: - & $156(84.8)$ & 39 & 117 & 75 & 0.686 \\
\hline HPV type 52 status at the time of HSIL diagnosis: + & $28(15.2)$ & 6 & 22 & 78.6 & \\
\hline HPV type 53 status at the time of HSIL diagnosis: - & $174(94.6)$ & 44 & 130 & 74.7 & 0.297 \\
\hline HPV type 53 status at the time of HSIL diagnosis: + & $10(5.4)$ & 1 & 9 & 90 & \\
\hline HPV type 56 status at the time of HSIL diagnosis: - & $164(89.1)$ & 37 & 127 & 77.4 & 0.093 \\
\hline HPV type 56 status at the time of HSIL diagnosis: + & $20(10.9)$ & 8 & 12 & 60 & \\
\hline HPV type 58 status at the time of HSIL diagnosis: - & $172(93.5)$ & 44 & 128 & 74.4 & 0.209 \\
\hline HPV type 58 status at the time of HSIL diagnosis: + & $12(6.5)$ & 1 & 11 & 91.7 & \\
\hline HPV type 59 status at the time of HSIL diagnosis: - & $173(94)$ & 43 & 130 & 75.1 & 0.620 \\
\hline HPV type 59 status at the time of HSIL diagnosis: + & $11(6)$ & 2 & 9 & 81.8 & \\
\hline HPV type 66 status at the time of HSIL diagnosis: - & $177(96.2)$ & 43 & 134 & 75.7 & 0.797 \\
\hline HPV type 66 status at the time of HSIL diagnosis: + & $7(3.8)$ & 2 & 5 & 71.4 & \\
\hline HPV type 67 status at the time of HSIL diagnosis: - & $177(96.2)$ & 41 & 136 & 76.8 & 0.058 \\
\hline HPV type 67 status at the time of HSIL diagnosis: + & $7(3.8)$ & 4 & 3 & 42.9 & \\
\hline HPV type 68 status at the time of HSIL diagnosis: - & $180(97.8)$ & 43 & 137 & 76.1 & 0.254 \\
\hline HPV type 68 status at the time of HSIL diagnosis: + & $4(2.2)$ & 2 & 2 & 50 & \\
\hline $\begin{array}{l}\text { Non-persistence of HPV infection at the time of HSIL diagnosis: The } \\
\text { present one and the same HPV type infection has not been present for at } \\
\text { least two years or absence of HPV infection }\end{array}$ & $19(10.3)$ & 8 & 11 & 57.9 & 0.113 \\
\hline $\begin{array}{l}\text { Persistence of HPV infection at the time of HSIL diagnosis: The present one } \\
\text { and the same HPV type infection has been present for at least two years }\end{array}$ & $23(12.5)$ & 6 & 17 & 73.9 & \\
\hline Persistence of HPV infection at the time of HSIL diagnosis: unknown & $142(77.2)$ & 31 & 111 & 78.2 & \\
\hline
\end{tabular}


Table 5. Statistical data of significant variables.

\begin{tabular}{|c|c|c|c|c|}
\hline & & Odds-ratio & $P$ value & $95 \%$ Confidence interval \\
\hline \multirow[t]{2}{*}{ All subjects } & $\bullet$ hrHPV & 6.97 & 0.008 & $1.67-29.17$ \\
\hline & - HPV16 & 2.79 & 0.004 & $1.38-5.66$ \\
\hline \multirow[t]{3}{*}{ Cohort one } & - hrHPV & 6.47 & 0.017 & $1.40-29.80$ \\
\hline & • HPV16 & 3.54 & 0.015 & $1.28-9.84$ \\
\hline & - HPV67 & 0.093 & 0.045 & $0.009-0.947$ \\
\hline Cohort two & - Persistent HPV infection & 1.17 & 0.029 & $1.02-1.35$ \\
\hline
\end{tabular}

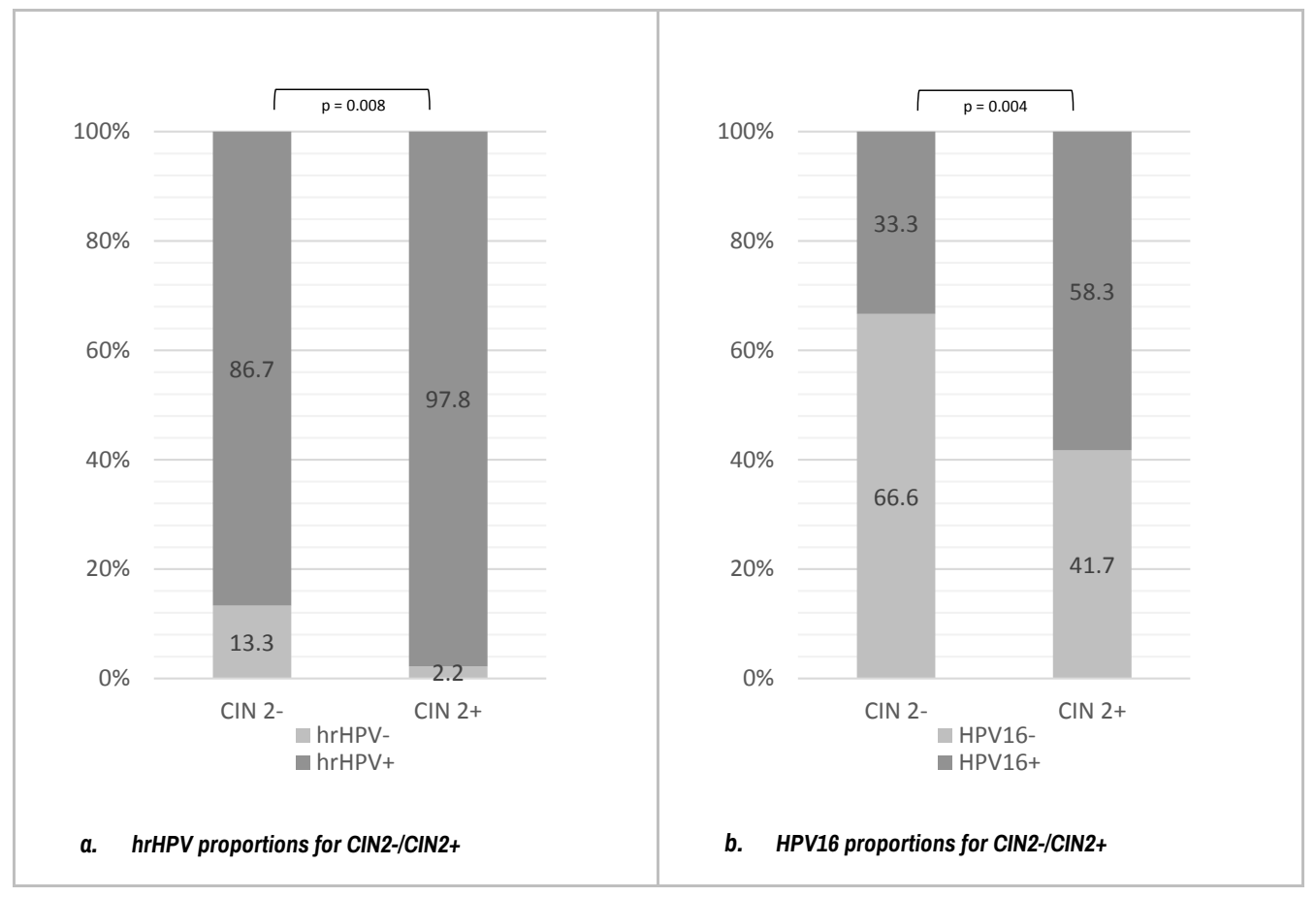

Figure 5. Significant variables (all included subjects).

Next, a logistic regression was performed on the subgroup of HPV16 positive women, to investigate the effect of more than one different hrHPV type infections. This showed to have no statistically significant effect $(p=0.5$, $\mathrm{OR}=1.26$ ).

\section{Statistical analysis of cohort one}

Analogous to the analysis of the whole group (5.5.1.), the variables hrHPV infection and HPV16 infection were statistically significant for the 91 included women from cohort one (TP, 2014) (Table 5). Those with a hrHPV infection at the time of HSIL diagnosis had 6.47 more chance (OR) to be $\mathrm{CIN2}^{+}$on histology than those who were hrHPV negative at that moment, with a $95 \% \mathrm{Cl}$ of $1.40-29.80$. This is statistically significant $(\mathrm{p}=0.017)$. Those with a HPV16 infection had 3.54 more chance to be CIN2 ${ }^{+}$than those who were HPV16 negative, with a $95 \% \mathrm{Cl}$ of $1.28-9.84(\mathrm{p}=0.015)$.

For this cohort an OR of 0.093 was found for the variable HPV67 infection, which would imply that a HPV67 infection at the time of HSIL diagnosis reduces the chance to be $\mathrm{CIN2}{ }^{+}$. Note that the $\mathrm{p}$-value is 0.045 and the $95 \% \mathrm{Cl}$ is large $(0.009-0.947)$ as in Table 5. one.

The other analyses variables were not statistically significant for cohort

For cohort one alone, the multivariate regression performed on the HPV types 16,31 and 67 , again showed that there is no confounding.

\section{Statistical analysis of cohort two}

For the 93 included women from cohort two (SP, 2010), the only variable found to be statistically significant $(p=0.029)$ was persistent HPV infection (Table 5). Women from cohort two had 1.17 more chance to be
CIN2 ${ }^{+}$when having a persistent HPV infection at the time of HSIL diagnosis (95\% Cl 1.02-1.35).

The other analysed variables were not statistically significant for cohort two. The multivariate regression performed on the HPV types 16,31 and 67 , again, showed no confounding.

\section{Discussion}

The percentage of HSIL-positive women with a histological CIN2 ${ }^{+}$result within one year is $75.5 \%(95 \% \mathrm{Cl}$ : $69.33-81.75)$ in AML, Antwerp. Comparing this to the result of $77.5 \%$ of our previously conducted systematic review [3], we find that there is only a difference of $2.0 \%$ between these two outcomes and that this difference is not significant as $77.5 \%$ lies within the $95 \% \mathrm{Cl}$. However, some notable differences are found between this study and the cited systematic review. A major distinction is the worldwide extent of the systematic review, in contrast to this study where only Flemish (North Belgium) women were included. Further, the review only discussed screening cytological samples, whereas this study discusses all collected samples (excluding GHAPRO and women without INSZ number) in AML without distinguishing between screening and diagnostic samples. Thus, in this study there is no knowledge of symptomatology of the included women. Also, the systematic review did not exclude women with histological followup after more than one year, in contrary to our defined inclusion criteria. Several articles that were included in the review have a study period of 5 years or more, which could lead to a higher detection rate of CIN2+. This could be a contributing factor for the slightly higher percentage found in the systematic review [3]. 
An important reason appointed in literature for the found false positivity rate of $24.5 \%$, is the use of $\mathrm{CDB}$. Multiple studies find that a higher detection rate of $\mathrm{CIN} 2^{+}$is observed when women undergo a LEEP or conisation, which are more reliable specimens [9-14]. Other variables like parity, menopausal status and use of oral contraceptive pills, are reasons for false positive results. Particularly HSIL positive nulliparous women, post-menopausal women and women who do not use oral contraceptive pills are found to have more CIN2- results on histology [9,14].

Next to the main aim of our study, it also seemed interesting to make an extra feature on the difference between the two cytological collection media used in AML over the past years (SurePath ${ }^{\mathrm{TM}}$ LBC and ThinPrep ${ }^{\circledR}$ $\mathrm{LBC})$. The found $\mathrm{CIN2}{ }^{+}$percentages for TP and SP, were $75.8 \%(95 \% \mathrm{Cl}$ 67.0-84.6) and $75.3 \%(95 \% \mathrm{Cl} 66.5-84.1)$ respectively. It is demonstrated that there is no significant difference between these two cytological techniques concerning outcome ( $p$-value $=0.930, \mathrm{OR}=1.03$ ). Still, to be precise, the statistical analyses were also made on both cohorts separately. In literature, we find similar results. Cox et al. indirectly compared the two liquid based cytology methods and did not detect a difference in sensitivity [15]. Also, Zhao et al. concluded that SurePath ${ }^{\mathrm{TM}}$ and ThinPrep ${ }^{\circledR}$ samples yielded analogous validity in detecting significant cervical lesions [16]. Yet, the rate of satisfactory slides was higher with use of SP, as the cell yield is larger because the brush of the collecting device is retained, contrary to TP, where the brush is rinsed and discarded $[16,17]$. Also, residual volumes for Hybrid Capture 2 (HC2) HPV DNA testing were larger when using SP [16]. However, we note that little literature was found specifically researching the difference between SP and TP LBC. More often, the focus lies on the comparison between conventional cytology and LBC.

Regarding the applied variables on the total group of 184 included women, the statistical analysis demonstrates that for women with a HSILpositive smear the variables hrHPV positivity and HPV16 positivity are significant for having a histological $\mathrm{CIN} 2^{+}$outcome within one year after cytological testing. The highest OR (6.97) is found for hrHPV-positivity, which implies that a HSIL positive woman who is also hrHPV positive has around seven times more risk for developing a histological CIN2+ lesion within one year than a HSIL positive woman who is hrHPV negative. For HPV16 positive women this risk is around three times higher $(\mathrm{OR}=2.79)$. HPV16 is the most carcinogenic type. This corresponds with literature, as Smelov et al. systematically reviewed risks of different HPV types for $\mathrm{CIN2}{ }^{+}$ and found that HPV genotype 16 contributed the greatest proportion of CIN2+ [18]. They also found that all hrHPV types together contributed $86.9 \%$ of CIN2+ lesions, which is analogous to our findings [18].

Persistence of HPV-infection was found to be a nonsignificant variable. There was a nonsignificant difference of $16 \%$ in $\mathrm{CIN}^{+}$percentage between the two subgroups "persistent infection for at least two years" and "no persistent infection". This is different from what is found in several articles $[19,20]$. One of the largest prospective screening trials with a followup period of over 13 years, Elfgren et al., found that all (40 of $40,100 \%$ [95\% $\mathrm{Cl}, 91-100 \%])$ patients who have persistent HPV-infection eventually will be diagnosed with $\mathrm{CIN} 2^{+}$, many in as few as two years. In this study a persistent HPV-infection is defined as an infection persisting over 12 months [19]. In our study we found that $73,9 \%$ (17 of 23) of women with a persistent HPV-infection, defined as an infection persisting over 2 years, developed a CIN2 ${ }^{+}$lesion. A reason for the lower percentage found in our study could be the small group of women who had one of the same HPV type infection for at least two years. It is important to highlight that of $77.2 \%$ of women included in our study the persistence of HPV-infection is unknown. The two main reasons for unknown persistence status are the following: the longest follow-up interval is less than two years (if the specific HPV-type was persistent at that moment), the shortest follow-up interval is more than two years (if the specific HPV-type was cleared at that moment). The large amount of 142 women (out of 184 women) with an unknown HPV-persistence can be explained by our set definition for persistence of 2 years. Across studies, it is clear that the definition of HPV persistence varies considerably [21]. Koshiol et al. conducted a systematic review regarding persistent HPV infections. We cite: "The minimum duration of HPV persistence was less than 6 months for 30 percent of studies, 6-12 months for $45 \%$, and more than 12 months for $25 \%$ " [21]. Another example is Sand et al. who defined persistence as having the same genotype twice 1-4.5 years apart [22].

The number of different hrHPV-types at the time of HSIL diagnosis was found to be a nonsignificant variable. There is little difference between the presence of one $(78.7 \%)$, two $(75.5 \%)$, or more than two (78.1\%) types. In literature we find that consensus is pending regarding the role of multiple hrHPV-strains in developing CIN2+ histology [23]. In Chaturvedi et al. coinfection with multiple high-risk species (more specifically HPV $16,31,33$, $35,52$ and 58$)$ resulted in a significantly increased risk of $\mathrm{CIN} 2^{+}(\mathrm{OR}=2.2$ $95 \% \mathrm{Cl}=1.1-4.6)$, compared with single HPV-infection [24]. In Li et al. on the other hand, they found that patients with single HPV16-infection had higher incidence of $\mathrm{CIN2}^{+}(62.2 \%)$ than patients with HPV16 infection mixed with other hrHPV-types (52.4\%) [25].

The age at time of HSIL-diagnosis was also found to be a nonsignificant variable. In our study we found that $77.5 \%$ of HSIL-positive women aged $<30$ years and $75 \%$ of HSIL-positive women aged 30 years or older have a histological $\mathrm{CIN} 2^{+}$lesion. This slightly higher percentage of younger women does not entirely correspond with the found literature on this subject. Einstein et al. states that cervical lesions found in women younger than 30 are rarely clinically significant [26]. Amongst young females who have experienced their first sexual intercourse there is a high HPV-infection rate. Winer et al reports a $29 \%$ one-year cumulative incidence of HPV-infection after first sexual introduction, increasing to $50 \%$ by three years [27]. In young women these infections are often transient, resulting in transient cytological lesions. A possible explanation for the higher percentage found in our study could be the fact that in this study no exclusive screening population was used and thus diagnostic smears could have been included.

It is noticeable that $100 \%$ of the HPV $45+$ women are CIN2 ${ }^{+}$, keeping in mind that this regards a very small group of only 3 women. Only $1.7 \%$ of the included HPV+ women harbored the HPV45 genotype. During statistical analysis no $p$-value could be calculated thus no conclusions could be made regarding the significance of this variable. In literature, a low prevalence of HPV45 infection is indeed found. In Peralta-Rodriguez et al., $0.82 \%$ of HSIL+ women (3 of 364) were HPV45 positive [28].

Regarding the applied variables on the two cohorts of 91 and 93 included women respectively, the statistical analysis demonstrates that for women from cohort one (TP, 2014) with a HSIL-positive smear the variables hrHPV-positivity and HPV16-positivity are significant for having a histological CIN2+ outcome within one year after cytological testing. On the contrary, HPV67-positivity appears to be a protective factor with an OR of 0.093 and $p$-value of 0.045 . It must be noted that the $95 \% \mathrm{Cl}$ of this low risk-HPV type is very large $(0.009-0.947)$ which implies that this is a coincidental finding. Also, no literature could be found that demonstrates a possible protective role of HPV67-infection for the development of highgrade cervical lesions.

For cohort two (SP, 2010) the only statistically significant variable found, was persistent HPV infection (OR 1.17). As mentioned above, this is congruent with the existing literature $[19,20]$.

In our study $76.7 \%$ had histological follow-up within one year. This percentage is lower than the percentages found in the 2018 report of the Flemish government concerning the cervical screening program "Jaarrapport 2018 Bevolkingsonderzoek baarmoederhalskanker" [2]. In this report only $5-10 \%$ of HSIL positive women have no histological follow-up within twelve months. However, it must be noted that this report concerns samples dated from 2016, whereas our included samples date from 2010 and 2014. As stated in the report, the follow-up rate has inclined from $73.0 \%$ in 2013 to $80.2 \%$ in 2016 (when looking at all abnormal cervical cytology results as one group, including HSIL) [2], which could explain the higher follow-up percentage found in 2016. In our study we found no statistically significant variable that influenced the follow-up rate.

Looking at the relevance of our study, theoretically it contributes to a 
more up-to-date scientific knowledge in de field of cervical screening in Flanders. Regarding the external validity of this study, we can point out that the found $\mathrm{CIN2} \%$ in AML, Antwerp is similar to the percentages found in worldwide laboratories. Thus, AML performs equally as good as other studied laboratories worldwide. Practically, based on our findings we could advice that in HSIL positive women who are HPV16 or hrHPV positive, extra attention should be paid to adequate anamnesis for alarm symptoms and timely histologic follow-up. Although, the results of our study are mostly practically relevant for cytopathologists, not general practitioners, because we only looked at HSIL+ women. Regarding consequences for public health, we should still be aware of the factor of overtreatment and the costs this brings to society.

\section{Conclusion}

The found $\mathrm{CIN2}^{+} / \mathrm{HSIL}$ ratio of $75.5 \%$ in $\mathrm{AML}$, Antwerp is similar to the percentages found in worldwide laboratories. HSIL positive women who are HPV16+ or hrHPV + are at significant higher risk for invasive cervical disease. No statistically significant difference in $\mathrm{CIN} 2+\%$ was found between the two LBC techniques TP and SP.

\section{Limitations and Recommendations for Future Studies}

A limitation of our study is the fact that we solely researched a homogenous group of HSIL positive women. No control group of HSIL negative women was enrolled, thus no sensitivity and specificity of cytology could be calculated. Also, the initial HSIL diagnosis was not reviewed by a second cytopathologist. A third limitation is the retrospective character of our study, as no fixed follow-up period could be set in the initial outline of the study. In a prospective cohort study loss to follow-up including missing values could be reduced.

However, with our study design we mimic a real-life situation yielding realistic data (e.g. non-compliance due to personal reasons), opposed to an artificially set up study. Other strengths of our study are working with the accredited laboratory "AML" with a large dataset (94.697 analysed cervical smears in 2014 and 89.761 in 2010), the comparison between TP and SP and the interpretation of these cohorts by the same pathologist. A last strength is the practice of HPV informed guided screening, a combination of serial co-testing and FPGS $[6,8,29]$.

More grounded clinical relevance will need to be proved in future larger studies. Another suggestion for further research is an adaptation of the study design with implementation of a control group (of HSIL negative women) and a prospective approach.

\section{Acknowledgements}

This study was initiated by Johannes Bogers, Professor in the Faculty of Cell Biology and Histology at the University of Antwerp and Medical Director of Pathology in AML, Laboratory of Molecular Pathology, Antwerp. We are grateful for the help of Ina Benoy, Medical Director of the Department of Molecular Diagnostics in AML.

\section{Conflict of Interest}

Authors NK and AVL declare that they have no conflict of interest. Authors IB and JB are both working in a laboratory processing cytological and molecular biology specimens (AML BVBA).

\section{References}

1. Cancer in an Ageing Population in Belgium 2004-2016, Belgian Cancer Registry, Brussels, 2018. Available from: https://kankerregister.org.
2. Jaarrapport Bevolkingsonderzoeken naar kanker 2018; Centrum voor Kankeropsporing- Belgian Cancer Registry, Brugge 2018. Available from: https://baarmoederhalskanker.bevolkingsonderzoek.be.

3. Karia Nina, Van Loon Alison, and Simoens Cindy "The positive predictive value of high-grade sqamous intraepithelial lesion on cytology for the histological diagnosis of cervical intraepithelial neoplasia 2 or higher: a systematic review." Acta Cytologica 4 (2019):1-9.

4. Agramunt, Sílvia, Miguel Ángel Checa, Mireia González and Larrazaba Fernando, et al. "High-grade squamous intraepithelial lesion could be managed conservatively in women up to 25 years: results from a retrospective cohort study." Journal of Lower Genital Tract Disease 17 (2013):459-462.

5. Qaseem, Amir, Patrick Alguire, Paul Dallas "Appropriate use of screening and diagnostic tests to foster high-value, cost-conscious care." Ann Intern Med 156 (2012):147-149.

6. Arbyn Marc, Depuydt Christophe, Benoy Ina, and Bogers Johannes, et al. "Valgent: A protocol for clinical validation of human papillomavirus assays." Journal of clinical virology: the official publication of the Pan American Society for Clinical Virology. 76 (2016): S14-21.

7. https://surveysystem.com

8. Bogers J. Focal point guided screening. (2008) Retrieved from http://www. belcyto.ulg.ac.be/education/presentations/061208Bogers.pdf

9. Numnum Michael, Kirby Tyler, and Leath Charles "A prospective evaluation of "see and treat" in women with HSIL Pap smear results: is this an appropriate strategy?" Journal of Lower Genital Tract Disease 9 (2005): 2-6.

10. Aue-Aungkul Apiwat, Punyawatanasin Sitthisack, Natprathan Apaporn, and Srisomboon Jatupol, et al. "See and treat approach is appropriate in women with high-grade lesions on either cervical cytology or colposcopy." Asian Pacific Journal of Cancer Prevention 12 (2011):1723-1726.

11. Boonlikit Sathone "Prevalence of high-grade cervical lesion in women with LSIL and HSIL cytology and prevalence of invasive cancer in women cytologically positive for malignancy." Asian Pacific Journal of Cancer Prevention 9 (2008): 715-718.

12. Kabaca C, Sariibrahim Bahar, Keleli I, and Karateke Ates et al. "The importance of immediate verification of a cervical cytological abnormality with histology." Indian Journal of Cancer 50 (2013): 292-296.

13. Kantathavorn Nuttavut, Phongnarisorn Chailert, Srisomboon Jatupol, and Suprasert Prapaporn, et al. "Northern Thai women with high grade squamous intraepithelial lesion on cervical cytology have high prevalence of underlying invasive carcinoma." Asian Pacific Journal of Cancer Prevention 7 (2006): 477-479.

14. Poomtavorn Yenrudee, Himakhun Wanwisa, Suwannarurk Komsun, and Thaweekul Yuthadej et al. "Cytohistologic discrepancy of high-grade squamous intraepithelial lesions in Papanicolaou smears." Asian Pacific Journal of Cancer Prevention 14 (2013): 599-602.

15. Cox Thomas "Liquid-based cytology: evaluation of effectiveness, costeffectiveness, and application to present practice." J Natl Compr Canc Netw 2 (2004): 597-611.

16. Zhao Fang-Hui, Hu Shang-Ying, and Bian Jessica "Comparison of ThinPrep and SurePath liquid-based cytology and subsequent human papillomavirus DNA testing in China." Cancer Cytopathol 119 (2011): 387-394.

17. Rozemeijer Kirsten, Naber Steffie and Penning Corine "Cervical cancer incidence after normal cytological sample in routine screening using SurePath, ThinPrep, and conventional cytology: population based study". BMJ 14 (2017): j504.

18. Smelov Vitaly, Elfstrom Miriam, and Johansson Anna "Long-term HPV typespecific risks of high-grade cervical intraepithelial lesions: A 14-year follow-up of a randomized primary HPV screening trial." Int. J. Cancer 136 (2015): 1171-1180.

19. Elfgren Kristina, Elfström Miriam, and Naucler Pontus "Management of women with human papillomavirus persistence: long-term follow-up of a randomized clinical trial." Am J Obstet Gynecol 261 (2017): 264.e1.

20. Einstein $\mathrm{MH}$ and Burk Robert "Persistent Human Papillomavirus Infection: definitions and clinical implications." Papillomavirus Report 12 (2001):119.

21. Koshiol Jill, Lindsay Lisa, and Pimenta Jaenna "Persistent Human Papillomavirus Infection and Cervical Neoplasia: A systematic Review and Meta-Analysis." Am J Epidemiol. 168 (2008):123-137. 
22. Sand Freja, Munk Christian, and Frederiksen Kirsten "Risk of CIN3 or worse with persistence of 13 individual oncogenic HPV types." Int J Cancer 144 (2019):1975-1982

23. De Brot Louise, Pellegrini Bruno, and Moretti Sabrina Teixeira "Infections with multiple high-risk HPV types are associated with high-grade and persistent lowgrade intraepithelial lesions of the cervix." Cancer Cytopathol 125 (2017):138-143.

24. Chaturvedi Anil, Katki Hormuzd, and Hildesheim Allan "A Human papillomavirus infection with multiple types: pattern of coinfection and risk of cervical disease." J Infect Dis. 203 (2011): 910-920.

25. Li Mingxia, Du Xinxin, and Lu Menghan "Prevalence characteristics of single and multiple HPV infections in women with cervical cancer and precancerous lesions in Beijing, China." J Med Virol 91 (2019): 473-481.

26. Einstein MH, Goff B, and Falk SJ "Human papillomavirus testing of the cervix: Management of abnormal results." (2017) Available from: www.uptodate.com.

27. Winer Rachel, Feng Qinghua, and Hughes James "Risk of female human papillomavirus acquisition associated with first male sex partner." $\mathrm{J}$ Infect Dis 197 (2008): 197-279.

28. Peralta-Rodriguez Raul, Romero-Morelos Pablo, and Villegas-Ruiz Vanessa "Prevalence of human papillomavirus in the cervical epithelium of Mexican women: meta-analysis." Infect Agent Cancer. 7 (2012): 34.

29. Valentine Kristi, Vanden Broeck Davy, Benoy lan, and Truyens Marie, et al "Cytology at the Time of HPV: Some Things to Think about when Discussing HPV." Acta cytologica. 60 (2016): 527-533.

How to cite this article: Nina Karia, Alison Van Loon, Ina Benoy and Johannes Bogers. What is the Retrospective Correlation between High-Grade Squamous Intraepithelial Lesion (HSIL) on Cytology and the Histological Diagnosis of Cervical Intraepithelial Neoplasia 2 (CIN2) or More in AML, Antwerp, Belgium? J Cytol Histol 10 (2020) doi: 10.37421/jch.2020.11.550 